\title{
The Influence of Clinical Staging and Use of Antiretroviral Therapy on Cognitive Functioning of School-Aged Nigerian Children with HIV Infection
}

\section{Gbemisola O Boyede ${ }^{1,2 *}$, Foluso E A Lesi ${ }^{2}$, Veronica C Ezeaka ${ }^{2}$ and Charles S Umeh ${ }^{3}$}

${ }^{1}$ Division of Developmental Paediatrics, School and Child and Adolescent Health, Red Cross War Memorial Children's Hospital, University of Cape Town, Cape Town, South Africa

${ }^{2}$ Department of Paediatrics, College of Medicine, University of Lagos, Nigeria

${ }^{3}$ Clinical Psychology Unit, Department of Psychiatry, Lagos University Teaching Hospital, Lagos, Nigeria

\begin{abstract}
Objective: To find out how the World Health Organization Clinical Stages of HIV infection and use of antiretroviral therapy influence cognitive functioning of school-aged HIV-infected Nigerian children.

Method: Cognitive assessments of 69 HIV positive children aged 6-15 years were performed using Raven's Standard Progressive Matrices. Thirty children were on Highly Active Antiretroviral Therapy. The children were subdivided for the purpose of analysis into two cognitive developmental stages using Piaget's developmental stage: the concrete operation stage (6-11 years) and the formal operation stage (12-15 years). Children on antiretrovira therapies were also compared with those not on therapies. HIV infection was staged using the revised World Health Organization Clinical Staging for Paediatric HIVIAIDS.

Result: The overall mean cognitive score for HIV positive subjects on antiretroviral therapy was 20.9 which was significantly higher than the overall score for subjects not on therapies of $14.6(p=0.007)$. In the age group $6-11$ years, the mean cognitive scores became lower significantly with worsening clinical stage for subjects irrespective of use of anti-retroviral therapy. The mean scores for subjects on therapy were significantly higher than that of their counterparts not yet on therapy at the same clinical stage. There were no significant differences in the cognitive scores at different clinical stages among the children in the age group 12-15 years.

Conclusion: Cognitive scores were lower with worsening clinical staging in the younger school-aged HIV-infected Nigerian children. Subjects on Highly Active Anti-retroviral Therapy had better cognitive scores than subjects not on therapy at the same clinical stage. Routine neuropsychological evaluation for school-aged HIV-infected children are recommended with an emphasiss on the younger children.
\end{abstract}

Keywords: Pediatric HIV; Cognitive assessment; School-age children; Ravens progressive matrices; Nigeria; WHO clinical staging; Antiretroviral therapy

Abbreviations: APIN: Aids Prevention in Nigeria; HAART: Highly Active Antiretroviral Therapy; PEPFAR: President's Emergency Plan for AIDS Relief; WHO: World Health Organization; RPM: Raven's Standard Progressive Matrices

\section{Introduction}

The Human Immunodeficiency Virus (HIV) infection and Acquired Immunodeficiency Syndrome (AIDS) is a global pandemic. Pediatric HIV/AIDS is a significant cause of childhood morbidity and mortality in Africa [1]. Without antiretroviral treatment, the progression of HIV infection in children is particularly aggressive, and many children die at a young age [2]. Nigeria is reported in 2009 to have an estimated 220,000 children less than 15 years old infected with HIV/AIDS and 1.2 million AIDS orphans [3]. Nigeria has the highest burden of motherto-child transmission (MTCT) rates and pediatric HIV disease in the world [4].

Involvement of the central nervous system (CNS) contributes substantially to morbidity and mortality of infection with the human immunodeficiency virus (HIV) [5]. The main neurological condition related to HIV infection in childhood is HIV-associated progressive encephalopathy, which may be the initial presenting condition for AIDS in $18 \%$ of cases. It affects between $30-60 \%$ of seropositive infants, children and adolescents at any point in time of their disease
[6]. Before Highly Active Antiretroviral Therapy (HAART) was introduced,encephalopathy was reported in up to $50 \%$ of children with a diagnosis of AIDS in the United States [7]. HIV-associated progressive encephalopathy causes neuropsychological deficits involving domains such as speech and language, memory, learning, information processing and motor functioning. They may affect negatively the children's normal development and school achievement [6].

However, advances in the medical treatment of children with HIV, including the development and use of combination antiretroviral therapies and supportive medications, not only have prolonged survival but also have promoted growth and development and have improved quality of life [8]. Despite these advances, many children

${ }^{*}$ Corresponding author: Gbemisola O Boyede, Division of Developmenta Paediatrics, School and Child and Adolescent Health, Red Cross War Memoria Children's Hospital, University of Cape Town, Cape Town, South Africa, Tel: +27791150729; E-mail: gbemisola.boyede@uct.ac.za

Received January 25, 2013; Accepted February 26, 2013; Published March 03 2013

Citation: Boyede GO, Lesi FEA, Ezeaka VC, Umeh CS (2013) The Influence of Clinical Staging and Use of Antiretroviral Therapy on Cognitive Functioning of SchoolAged Nigerian Children with HIV Infection. J AIDS Clin Res 4: 195. doi:10.4172/21556113.1000195

Copyright: (c) 2013 Boyede GO, et al. This is an open-access article distributed under the terms of the Creative Commons Attribution License, which permits unrestricted use, distribution, and reproduction in any medium, provided the original author and source are credited. 
still experience direct and indirect effects of HIV infection that affect their experienceas effective learners. Although use of antiretroviral drugs to treat children has increased in recent years in sub-Saharan Africa, children living with HIV are about one third as likely to receive antiretroviral therapy as adults living with HIV as a whole [9]. Additionally, developmental assessments are also not part of routine care in most HIV clinics in Africa despite the well-documented impact of HIV on neurodevelopment of affected children.

There is need for more research among African children beyond the preschool-age period. Many children are now surviving to school age in Africa due to free antiretroviral therapy in many countries including Nigeria. The impact of HIV on their cognitive function and school performance need to be evaluated. The influence of clinical staging and use of antiretroviral therapy in the cognitive functioning also need to be explored. Many clinicians depend on clinical staging in resource-poor setting in Africa in making decision about management of HIV-infected children especially the commencement of antiretroviral therapy. HIVinfected children in Nigeria are managed according to the National Guidelines for paediatric HIV/AIDS treatment and care based on the clinical and immunological stage of their disease [4]. The Guidelines are based on the current WHO Guidelines for the management of Paediatric HIV/AIDS [10]. The current study aimed to evaluate the cognitive functioning of HIV-infected school-aged Nigerian children receiving standard care (including antiretroviral therapy) at different WHO clinical stages. The study also compares cognitive scores of HIV-infected children on HAART with that of their HAART-naïve counterparts at the same clinical stage.

\section{Materials and Methods}

\section{Study location}

The study was conducted between November 2009 and April 2010 at Lagos University Teaching Hospital (LUTH), Lagos State, Nigeria. LUTH is a 750-bedded tertiary center providing health care to the inhabitants of Lagos and neighboring states. Children below the age of eighteen years have health care provided through the Department of Paediatrics at LUTH. Services provided include emergency pediatric services, inpatient care and specialty outpatient clinics which include a dedicated Paediatric HIV Clinic for HIV-infected and HIV-exposed children.

\section{Participants}

HIV-infected children at school-age (6-15 years) accessing care at Paediatric HIV Clinic, LUTH constituted the study population. The total number of children (comprising of infants perinatally exposed to HIV and HIV-infected children) attending the clinic at the end of 2009 was 566 out of which 223 were within the age group 6-15 years. The latter group had 174 children on HAART while 49 were not on HAART. The Clinic is being funded by the USA government through the President's Emergency Plan for AIDS Relief (PEPFAR). Human Immunodeficiency Virus infection was diagnosed with a positive Enzyme Linked Immunosorbent Assay (ELISA) test, which was confirmed with the Western blot test. HIV-infected children who presented at age below eighteen months had HIV diagnosis confirmed with the HIV DNA PCR tests [4].

\section{Inclusion criteria:}

1. Confirmed HIV positive status

2. Age 6-15 years inclusive
3. HIV status disclosure (partially or fully) to the child by the parents and/or chancellors and signed, informed consent of the parents/guardians as well as the assent (verbal/written) with the children.

\section{The exclusion criteria:}

1. Acute illness and/or hospital admission at the time of cognitive test administration,

2. Chronic neurological disorders such as epilepsy, cerebral palsy;

3. Inability to perform the RPM cognitive test for whatever reasons.

\section{Instrument}

The Raven's Progressive Matrices (RPM) is a widely used nonverbal test of general intelligence that appraises visuo-spatial reasoning, abstract thinking, deductive reasoning and general intelligence [11]. RPM has been validated for use in Nigerian children [12]. In RPM, a person is shown a matrix of patterns with one pattern missing. The person must figure out the rules governing the patterns and then use these rules to pick the item that best fills in the missing pattern. The test was designed to minimize the influence of culture by relying on nonverbal problems that require abstract reasoning and do not require knowledge of a particular culture. The RPM consists of sixty problems divided into five subsets of twelve. Each person's total score is the total number he/she got correctly. It is a culture-fair measure of cognitive functioning which is suitable for this kind of study.

\section{Procedure and data collection}

Consecutive HIV positive children attending the Paediatric HIV Clinic who satisfied the inclusion criteria were recruited until the desired sample size was attained. The minimum sample size required was calculated using a statistical formula (power-based calculation) [13]. After obtaining informed consents/assents from the parents/ children, the researcher administered the study Proforma. The Proforma consisted of five sections: (1) socio-demographic data (2) medical history (3) neurological symptoms (4) neurological examination and (5) RPM cognitive tests. The socioeconomic status of the family of the subjects was computed using the method recommended by Oyedeji [14]. HIV positive children had their disease staged using the WHO clinical staging [15].

\section{Ethical approval}

The study was approved by the LUTH Research and Ethics Committee before the commencement of the study. Informed, written consents were obtained from the parents/guardians and verbal/ written assents were obtained from the participants. The children were informed of their scores and parents/caregivers were counselled on need for follow-up care.

\section{Data analysis}

Data was entered, validated and analyzed using the SPSS for windows version 16.0 [16]. The means of continuous variables were compared using either the Student's $t$-test or ANOVA. A p value less than 0.05 was accepted as statistically significant (two-tailed analysis).

\section{Results}

Clinical and socio-demographic characteristics of HIV positive subjects

Sixty nine HIV positive children were recruited into the study; 39 
(57\%) were on HAART and $30(43 \%)$ were not. Table 1 showed the clinical and socio-demographic characteristics of the study subjects. The mean age at diagnosis of HIV for subjects on HAART was $4.85 \pm$ 3.28 years which were significantly higher than that of subjects not on HAART of $7.29 \pm 3.27$ years $(\mathrm{p}=0.01)$. The mean duration of treatment

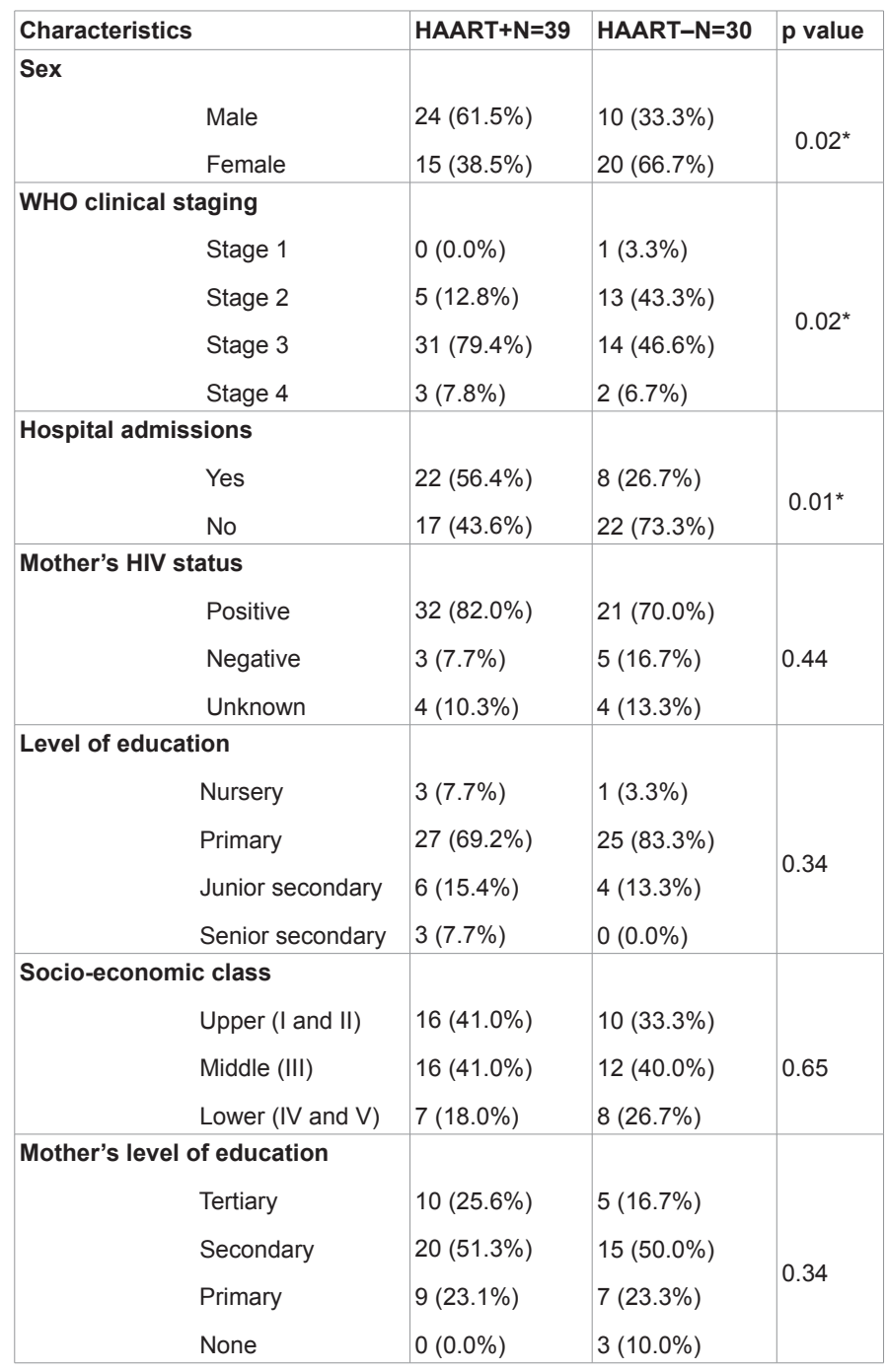

\section{* $p$ is significant}

\section{HAART+=children on HAART}

HAART -=children not on HAART

Table 1: Clinical and socio-demographic characteristics of the HIV positive children on HAART and those not on HAART. for HIV positive children on HAART was $48.13 \pm 23.43$ months. The HIV-infected subjects on HAART were comparable to subjects not on HAART in terms of age, maternal HIV status, level of education, socioeconomic class and maternal level of education. However, on gender distribution, there were more males (61.5\%) among subjects on HAART compared to subjects not on HAART (33.3\%). This difference was statistically significant $(\mathrm{p}=0.02)$. In respect of WHO clinical staging, $87 \%$ of HIV positive subjects on HAART were in WHO Stages 3 and 4 compared to $43.3 \%$ of subjects not on HAART at the same stages. This difference was also statistically significant $(\mathrm{p}=0.02)$.

\section{RPM scores of subjects on HAART and those not on HAART}

The overall mean RPM score for HIV positive subjects on HAART was 20.9 which was significantly higher than the mean for HAARTnaive subjects of $14.6(\mathrm{p}=0.007)$. Among the children in the age group 6-11 years, the mean RPM score of the HIV positive subjects on HAART was 17.1, which was significantly higher than the mean RPM score of 13.4 for HIV positive subjects who were not on HAART $(\mathrm{p}=0.046)$. For the age group 12-15 years, the mean RPM score of the HIV positive subjects on HAART was 33.4 which was also higher than that for subjects not on HAART of 20.4 but the difference failed to reach statistical significance $(\mathrm{p}=0.059)$.

\section{RPM scores and WHO clinical staging of subjects on HAART and not on HAART}

Among the HIV-infected children in the age group 6-11 years, the mean RPM scores were significantly lower with worsening WHO clinical stage for subjects on HAART $(\mathrm{p}=0.03)$. The trend was also similar for subjects not on HAART $(\mathrm{p}=0.001)$ as shown in Table 2. When the means at each WHO Clinical stage were compared, subjects on HAART had significantly higher means scores than subjects not on HAART at WHO Clinical Stage 2 and 3. No difference was observed in WHO stage 1 and 4.

In the age group 12-15 years, there was no significant difference in the mean scores across the WHO Clinical stages and between subjects on HAART and those not on HAART. This may be because there are few subjects in the age group which did not permit meaningful statistical analysis.

\section{Discussion}

The HIV positive children on HAART were comparable to the HAART-naive subjects in the majority of the clinical and socialdemographic characteristics except in terms of gender, age at diagnosis, WHO clinical staging and previous hospital admissions. The reason for the significantly higher female to male ratio among the HIV positive HAART-naive compared to the HIV subjects on HAART could not be

\begin{tabular}{|c|c|c|c|c|c|c|}
\hline \multirow{3}{*}{$\begin{array}{l}\text { WHO } \\
\text { STAGE }\end{array}$} & \multicolumn{4}{|c|}{ RPM SCORES } & \multirow{3}{*}{$t$ value } & \multirow{3}{*}{$p$ value } \\
\hline & \multicolumn{2}{|c|}{ ON HAART } & \multicolumn{2}{|c|}{ NOT ON HAART } & & \\
\hline & n (\%) & Mean \pm SD & n (\%) & Mean \pm SD & & \\
\hline STAGE 1 & $0(0.0 \%)$ & 0 & $1(4.0 \%)$ & 30.00 & - & - \\
\hline STAGE 2 & $5(16.7 \%)$ & $25.20 \pm 14.06$ & $12(48.0 \%)$ & $13.25 \pm 4.07$ & 2.79 & $0.01^{*}$ \\
\hline STAGE 3 & $23(76.7 \%)$ & $15.74 \pm 5.34$ & $11(44.0 \%)$ & $12.18 \pm 2.13$ & 2.10 & $0.04^{*}$ \\
\hline STAGE 4 & $2(6.7 \%)$ & $13.00 \pm 1.41$ & $1(4.0 \%)$ & 13.00 & 0.00 & 1.00 \\
\hline \multirow[t]{2}{*}{ Total } & $30(100.0 \%)$ & $17.13 \pm 7.93$ & $25(100.0 \%)$ & $13.44 \pm 4.69$ & 2.05 & $0.046^{*}$ \\
\hline & \multicolumn{2}{|c|}{$\begin{array}{c}F=3.84 \\
p=0.03^{*}\end{array}$} & \multicolumn{2}{|c|}{$\begin{array}{c}F=8.67 \\
p=0.001^{*}\end{array}$} & & \\
\hline
\end{tabular}

${ }^{*} p$ value significant

Table 2: RPM scores and WHO Clinical Stages of HIV positive children aged 6-11 years on HAART and not on HAART. 
explained. The differences in terms of age at diagnosis, WHO clinical stages and hospital admissions, however, were expected as the children on HAART were more likely to have been diagnosed earlier; have been admitted to the hospital at least once and belonged to the advanced WHO clinical staging with higher disease burden. This is a reflection of the fact that they have a more advanced illness.

The present study compared cognitive function across the different WHO clinical stages. In the age group $6-11$ years, the RPM scores tended to be lower with worsening WHO clinical stage irrespective of use of HAART. This finding is similar to that observed by Smith et al. [17] in USA in early school-aged and preschool-aged (3-7 years) children. The latter study used the Centre for Disease Control and Prevention (CDC) classification [18]. Children in the late WHO stages 3 and 4 have AIDS defining illnesses comparable to the advanced and severe immunosuppression stage $\mathrm{C}$ of the CDC classification $[15,18]$. The authors showed that children with HIV infection and class C status scored significantly lower in all domains of cognitive development using the McCarthy Scales; across all time points, than did those who were HIV infected without an AIDS-defining illness and those who were HIV exposed but not infected. All children in the US study were on antiretroviral therapy. This is comparable to our finding of lower cognitive scores among children in the late WHO clinical stages amongst children who were already on antiretroviral drugs. The finding of poorer cognitive functioning in this group of HIV-infected children is as a result of the HIV progressive encephalopathy which is more common in the later stages of HIV disease and may be the initial presenting condition of AIDS [6].

The finding of the better cognitive performance by HIV positive subjects on HAART compared to those not on HAART was similar to that by workers in USA and Argentina who documented remarkable improvement in HIV-related encephalopathy following commencement of antiretroviral therapy in infected children $[6,19]$. HAART reduces CSF viral load and the effect of the HIV on the brain, thus halting or reversing already established HIV encephalopathy [8]. In contrast, the neurological and cognitive deficits observed in a sample of HAARTnaive South African children at baseline remained the same after six months on HAART [20]. There was neither spontaneous resolution nor deterioration in the cognitive function of the study subjects. Lack of deterioration perhaps, showed beneficial effect of HAART at least in halting further cognitive decline. The HAART therapy of six months in the South African study is perhaps too short, compared to the mean duration on HAART therapy of 48 months in the present study, for the effect of HAART on cognitive performance to be appreciated. The implication of the finding of better cognitive functioning among HIV positive subjects on HAART in this study further justifies the need to commence all HIV positive children on antiretroviral therapy. This is not yet routine practice in many African countries despite recent $\mathrm{WHO}$ recommendations [10].

In Nigeria, all children below the age of two years are commenced on antiretroviral therapy. The finding of this study is suggesting that HIV-infected children at school age who currently do not qualify for ARV based on clinical and immune status according to the National Guidelines; may need ARV if they develop HIV-associated cognitive impairment despite being clinically asymptomatic. However, cognitive assessments are not yet part of routine care in HIV clinics in the country. This study is highly recommended that while longitudinal multi-centered studies are being planned to influence policy change especially as regards antiretroviral therapy, HIV-infected children in Nigeria and other African countries should have routine cognitive assessments as part of holistic care.
The current study has some limitations. It was a cross-sectional study with just one cognitive assessment. A longitudinal cohort study with multiple interval assessments would have given more information about cognitive deficits over time. Additionally, different domains of cognitive function were not assessed. This is because of the need to perform a brief cognitive test that can be done on an outpatient basis. A longitudinal follow-up cohort study is recommended that will incorporate interval assessments to evaluate cognitive function over time to further confirm the findings of this preliminary study. Neurocognitive assessment of a larger sample of children in the age group 12-15 years will also be needed to further evaluate observed differences in cognitive performance on RPM between subjects on HAART and those not on HAART.

\section{Conclusion}

This study has shown that the cognitive RPM scores of school-aged HIV-infected Nigerian children in the age group of 6-11 years tended to become poorer as the WHO clinical stage worsened. Also HIV positive subjects on HAART had better cognitive functioning on the RPM than subjects not on HAART in the age group 6-11 years showing the positive mitigating effect of antiretroviral drugs on cognitive impairment in HIV/AIDS. It is recommended that all HIV positive Nigerian children should have a routine neuropsychological evaluation at intervals as part of their standard care to promptly detect children with cognitive impairment for early interventions.

\section{Acknowledgement}

We thank all the parents and children attending Paediatric HIV (APIN) who participated in this study. We also acknowledge the laboratory and data room staff of APIN clinic for their assistance during the course of the study.

\section{References}

1. Eley BS, Tindyebwa D, Kayita J, Kieffer MP, Nduati R, et al. (2006) Contextualising the paediatric HIV epidemic: a review. East Afr Med J 83: 684688.

2. Newell ML, Coovadia H, Cortina-Borja M, Rollins N, Gaillard P, et al. (2004) Mortality of infected and uninfected infants born to HIV-infected mothers in Africa: a pooled analysis. Lancet 364: 1236-1243.

3. Joint United Nation Programme on HIVIAIDS (2009) AIDS epidemic update. UNAIDS.

4. Federal Ministry of Health (2007) National Guidelines for Paediatric HIV and AIDS Treatment and Care. Federal Ministry of Health, HIV and AIDS Division, Abuja.

5. Exhenry C, Nadal D (1996) Vertical human immunodeficiency virus-1 infection involvement of the central nervous system and treatment. Eur J Pediatr 155 839-850.

6. Millana-Cuevas LC, Portellano JA, Martinez-Arias R (2007) Neuropsychologica impairment in human immunodeficiency virus-positive children. Rev Neurol 44 366-374.

7. Cooper ER, Hanson C, Diaz C, Mendez H, Abboud R, et al. (1998) Encephalopathy and progression of human immunodeficiency virus disease in a cohort of children with perinatally acquired human immunodeficiency virus infection. Women and Infants Transmission Study Group. J Pediatr 132: 808812

8. Chiriboga CA, Fleishman S, Champion S, Gaye-Robinson L, Abrams EJ (2005) Incidence and prevalence of HIV encephalopathy in children with HIV infection receiving highly active anti-retroviral therapy (HAART). J Pediatr 146: 402-407.

9. Prendergast A, Tudor-Williams G, Jeena P, Burchett S, Goulder P (2007) International perspectives, progress, and future challenges of paediatric HIV infection. Lancet 370: 68-80.

10. World Health Organization (2010) Antiretroviral therapy of HIV infection in infants and children: towards universal access. WHO, Geneva, Switzerland.

11. Raven JC (1958) Standard Progressive Matrices. Lewis HK, London. 
Citation: Boyede GO, Lesi FEA, Ezeaka VC, Umeh CS (2013) The Influence of Clinical Staging and Use of Antiretroviral Therapy on Cognitive Functioning of School-Aged Nigerian Children with HIV Infection. J AIDS Clin Res 4: 195. doi:10.4172/2155-6113.1000195

12. Ogunlade O (1978) The Predictive Validity of the Raven Progressive Matrices with Some Nigerian Children. Educ Psychol Meas 38: 465-467

13. Rosie C (2006) Statistics: An introduction to sample size calculations (online). Mathematics Learning Support Centre.

14. Oyedeji GA (1985) Socioeconomic and cultural background of hospitalized children in Ilesa. Nig J Paediatr 12: 111-117.

15. World Health Organization (2006) Revised WHO Paediatric Clinical Staging System. WHO, Geneva, Switzerland.

16. SPSS for windows (2007). Release 16.0.1. SPSS Inc, Chicago, USA.

17. Smith R, Malee K, Leighty R, Brouwers P, Mellins C, et al. (2006) Effects of perinatal HIV infection and associated risk factors on cognitive development among young children. Pediatrics 117: 851-862.

18. Centres for Disease Control and Prevention (1994) 1994 Revised classification system for human immunodeficiency virus infection in children less than 13 years of age. MMWR 43:1-10

19. Diamond GW, Gurdin P, Wiznia AA, Belman AL, Rubinstein A, et al. (1990) Effects of congenital HIV infection on neurodevelopmental status of babies in foster care. Dev Med Child Neurol 32: 999-1004.

20. Smith L, Adnams C, Eley B (2008) Neurological and neurocognitive function of HIV-infected children commenced on antiretroviral therapy. S Afr J Child hlth 2: 108-113. 